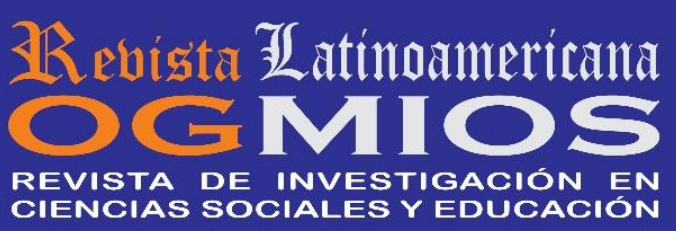

\title{
LA RETROALIMENTACIÓN EN EL PROCESO DE ENSEÑANZA - APRENDIZAJE DEL INGLÉS EN ESTUDIANTES DE SECUNDARIA
}

\section{FEEDBACK IN THE TEACHING PROCESS - LEARNING ENGLISH IN HIGH SCHOOL STUDENTS}

\author{
DOI: https://doi.org/10.53595/rlo.v1.i2.013
}

\author{
Isaias Jossue Olivera Sagua \\ Universidad Nacional del Altiplano \\ isaac7olivera@gmail.com
}

\section{RESUMEN}

La presente investigación tuvo como objetivo determinar la frecuencia en la que se emplea la retroalimentación como parte del proceso de enseñanza - aprendizaje del inglés en estudiantes de la institución educativa secundaria “José Carlos Mariátegui” Aplicación de la Universidad Nacional del Altiplano - Puno en el año 2019. La población de estudio estuvo constituida por 340 estudiantes del primero al quinto grado, secciones " $A$ " y " $B$ ". La muestra para esta investigación se determinó, a través del muestreo estratificado, resultando un total de 139 estudiantes. El tipo de investigación fue descriptivo diagnóstico con un diseño no experimental de enfoque cuantitativo. La técnica de investigación utilizada fue la encuesta y el instrumento, el cuestionario que mide la frecuencia con la que se emplea la retroalimentación. Los resultados demuestran que la retroalimentación por descubrimiento o reflexión es la más utilizada con un 53.2\%, es decir, los docentes del área de inglés crean espacios donde los estudiantes reflexionan acerca de su aprendizaje para descubrir sus errores y mejorarlos. Finalmente, se ha concluido que, la frecuencia con la que se emplea la retroalimentación se ubica en la escala de "Algunas veces", con una media aritmética de 29.7 puntos.

Palabras clave: Descubrimiento, reflexión, retroalimentación, proceso de enseñanza - aprendizaje.

\section{ABSTRACT}

The present research aimed to determine the frequency in which feedback is used as part of the teaching process - learning English in students of the secondary educational institution "José Carlos Mariátegui" Application of the National University of the Altiplano - Puno in the year 2019. The study population consisted of 340 students from first to fifth grade, sections "A" and "B". The sample for this research was determined, through stratified sampling, resulting in a total of 139 students. The type of research was descriptive - diagnostic with a non-experimental design of quantitative approach. The research technique used was the survey and the instrument, the questionnaire that 
measures how often feedback is used. The results show that feedback by discovery or reflection is the most used with $53.2 \%$, that is, teachers in the English area create spaces where students reflect on their learning to discover their mistakes and improve them. Finally, it has been concluded that the frequency with which feedback is used is located on the scale of "Sometimes", with an arithmetic mean of 29.7 points.

Keywords: Discovery, reflection, feedback, teaching-learning process.

\section{INTRODUCCIÓN}

La enseñanza del inglés es un reto que todos los docentes del área tienen, en donde se pretende que los estudiantes desarrollen competencias comunicativas, es decir, aprendan y sepan comunicarse competentemente en interacciones con sus semejantes, en contextos diversos y/o determinados, en el idioma inglés como lengua extranjera ya sea interactuando con interlocutores o personas anglohablantes, al establecer diálogos, expresando ideas, sentimientos y emociones, escribiendo textos diversos e informando o narrándolos oralmente.

Para lograr estos propósitos existen estrategias diversas, métodos, técnicas, medios y materiales que pueden emplearse, sin embargo, la utilización de estos recursos no pueden ser suficientes, en efecto, la utilización de la retroalimentación debe ser un factor indispensable que no puede faltar en el proceso de enseñanza - aprendizaje del inglés que, también, contribuye significativamente en lograr estos propósitos comunicativos. Al respecto, Canabal y Margalef (2017) sostienen que la retroalimentación puede mejorar y motivar los aprendizajes posteriores de los estudiantes.

En muchas de las instituciones educativas del nivel secundario los estudiantes muestran desinterés, tedio y falta de motivación en aprender una lengua extranjera (inglés), que repercute significativamente en su proceso de aprendizaje, los factores que afectan dicho proceso pueden ser diversos, en tanto, estos pueden surgir del mismo estudiante y del docente quien tiene la responsabilidad de crear un ambiente favorable, empático y, sobre todo, comprensible. Estudios realizados a nivel mundial han demostrado que el estudiante ya no es un simple receptor de información sino aquel que gestiona la información, es sabido que a nivel nacional y local el Ministerio de Educación del Perú, viene priorizando la evaluación formativa en estudiantes, para lo cual, la retroalimentación es uno de los factores indispensables que no debe faltar en el proceso de enseñanza-aprendizaje. Un estudio realizado por Calvo (2018) demuestra que la mayoría de los docentes que son el $55 \%$, no utilizan la retroalimentación. 
Por otro lado, para comunicarse competentemente en inglés, los estudiantes deben saber manejar cuatro habilidades fundamentales (speaking, listening, writing and reading), sin embargo, en muchos de los casos no se logran manejar estas cuatro habilidades, generalmente éstos refieren que en las clases de inglés no consiguen comprender las explicaciones del docente, otros aluden que los docentes desarrollan su clase sin percatarse que los estudiantes estén asimilando el inglés. En efecto, en otra investigación realizada por Beltran (2017) señala que la retroalimentación de la evaluación no es concebida ni aplicada de una forma correcta. Es decir, que los docentes no están aplicando la retroalimentación como tal y más aún no se esté dando retroalimentación de calidad (por reflexión o descubrimiento). Desde estas perspectivas se asume y surge el problema en que los docentes no estén realizando una evaluación formativa durante el desarrollo de la sesión de clase, en otras palabras, no se esté evaluando para brindar una retroalimentación a los estudiantes o simplemente no se evalúa para retroalimentar. Se sabe que la adquisición de una lengua extranjera para muchos resulta complicada y mientras más no entendamos, más hostil será el proceso de aprendizaje, por lo que es indispensable evaluar y retroalimentar durante la sesión de clase. Al respecto, el MINEDU (2016) refiere que una retroalimentación por descubrimiento o reflexión es la más ideal que una descriptiva y elemental. Una evaluación para generar retroalimentación es cuando el docente realiza un acompañamiento del proceso de aprendizaje de los estudiantes durante la sesión de aprendizaje, ya sea mediante la evaluación formativa o monitoreando los avances y dificultades de aprendizaje, y cómo es que se brinda apoyo pedagógico pertinente a través de la retroalimentación, siempre y cuando se identifique las necesidades de aprendizaje de los estudiantes. En fin, según lo expuesto, se destaca la relevancia en la retroalimentación y más aún en la calidad de retroalimentación (por descubrimiento o reflexión) que se debe realizar para la enseñanza de un idioma extranjero.

El objetivo de esta investigación consiste en determinar la frecuencia en la que se emplea la retroalimentación como parte del proceso de enseñanza - aprendizaje del inglés en estudiantes de la Institución Educativa Secundaria “José Carlos Mariátegui” Aplicación de la UNA - Puno en el año 2019. La hipótesis señala que la frecuencia en la que se emplea la retroalimentación como parte del proceso de enseñanza - aprendizaje del inglés es "Algunas veces" en estudiantes de la Institución Educativa Secundaria "José Carlos Mariátegui" Aplicación de la UNA - Puno en el año 2019.

\section{MATERIALES Y MÉTODOS}




\section{Lugar de estudio}

El estudio se ejecutó en la ciudad, provincia y distrito de Puno - Perú. Concretamente el lugar donde se realizó la investigación fue en la institución educativa secundaria "José Carlos Mariátegui - Aplicación” de la Universidad Nacional del Altiplano - Puno, ubicado en el Jr. Jorge Basadre con las coordenadas -15.827820, -70.016829. El periodo de estudio corresponde al año 2019.

\section{Descripción de métodos}

Según el propósito de estudio, le corresponde el tipo básico - diagnóstico. Corresponde a este tipo porque los resultados de investigación permitirán incrementar la teoría existente sobre el tema.

Según la estrategia de investigación, corresponde a las investigaciones no experimentales cuantitativas, porque no se manipula ninguna variable ni se preparan las condiciones de investigación, es decir, los datos se recogieron tal como se presenta en la realidad (Charaja, 2011).

Además, Hernández, et al (2014) señalan que el enfoque cuantitativo permite la medición numérica y el análisis estadístico de las variables, para caracterizar y describirlos. Asi mismo, refieren que el tipo no-experimental, no involucra la experimentación de alternativas resolutivas a la problemática observada.

Para esta investigación se utilizó la técnica de la encuesta, además se empleó como instrumento el cuestionario, denominado "Cuestionario la retroalimentación”, el cual, permitió analizar y medir la variable de estudio. El "Cuestionario la retroalimentación" tiene un total de 15 preguntas agrupadas en tres grupos de cinco cada uno, en efecto, cada apartado obedece y permite medir las dimensiones: La retroalimentación por descubrimiento o reflexión, la retroalimentación descriptiva y/o adaptativa y la retroalimentación elemental. Para la organización y el tratamiento de datos se utilizó el programa estadístico SPSS versión 22 (software de análisis predictivo) y el Microsoft Excel.

\section{RESULTADOS Y DISCUSIÓN}

Los resultados que se muestran a continuación se presentan partiendo del análisis general de la variable de estudio (la retroalimentación) y/u objetivo general, seguido del análisis de las dimensiones de estudio y/u objetivos específicos. Estos resultados están agrupados, organizados y establecidos en tablas y figuras, debidamente analizadas e interpretadas. como profesor - alumno. 
Tabla 1

La retroalimentación en la enseñanza - aprendizaje del inglés en estudiantes de la I.E.S. “José Carlos Mariátegui” Aplicación de la UNA - Puno en el año 2019

\begin{tabular}{ccccc}
\hline Valoración & Frecuencia & Porcentaje & Porcentaje válido & $\begin{array}{c}\text { Porcentaje } \\
\text { acumulado }\end{array}$ \\
\hline Nunca & 4 & 2.9 & 2.9 & 2.9 \\
Algunas veces & 73 & 52.5 & 52.5 & 55.4 \\
Siempre & 62 & 44.6 & 44.6 & 100.0 \\
Total & 139 & 100.0 & 100.0 & \\
\hline
\end{tabular}

De acuerdo con los datos mostrados en la tabla 1, se puede decir que, de un total de 139 encuestas aplicadas a estudiantes que representan el 100\%, 4 de estas que equivale al $2.9 \%$ acreditan que la frecuencia en la que se emplea la retroalimentación como parte del proceso de enseñanza - aprendizaje del inglés, es "Nunca". Por otro lado, 73 encuestan aplicadas que equivalen el 52.5\% demuestran que la frecuencia en la que se emplea la retroalimentación, es "Algunas veces", y el $44.6 \%$ de 62 encuestas evidencian que la retroalimentación se da "Siempre".

Se observa que la mayor frecuencia en la que se emplea la retroalimentación como parte del proceso de enseñanza - aprendizaje del inglés es "Algunas veces", con un total de 73 encuestas que equivalen al $52.5 \%$ lo que significa que la retroalimentación, ya sea por descubrimiento o reflexión, descriptiva y/o adaptativa y elemental se da al menos una vez durante las sesiones de aprendizaje en estudiantes de la I.E.S. "José Carlos Mariátegui" Aplicación de la UNA - Puno en el año 2019

\section{Tabla 2}

La retroalimentación por descubrimiento o reflexión en la enseñanza - aprendizaje del inglés en estudiantes de la I.E.S. "José Carlos Mariátegui” Aplicación de la UNA - Puno en el año 2019

\begin{tabular}{ccccc}
\hline Valoración & Frecuencia & Porcentaje & Porcentaje válido & $\begin{array}{c}\text { Porcentaje } \\
\text { acumulado }\end{array}$ \\
\hline Nunca & 4 & 2.9 & 2.9 & 2.9 \\
Algunas veces & 61 & 43.9 & 43.9 & 46.8 \\
Siempre & 74 & 53.2 & 53.2 & 100.0 \\
Total & 139 & 100.0 & 100.0 & \\
\hline
\end{tabular}

De acuerdo con los datos mostrados en la tabla 2, se puede decir que, de un total de 139 encuestas aplicadas a estudiantes que representan el 100\%, 4 de estas que equivale al $2.9 \%$ acreditan que la frecuencia en la que se emplea la retroalimentación por descubrimiento o reflexión como parte del proceso de enseñanza - aprendizaje del inglés, es "Nunca". Por otro lado, 61 encuestan aplicadas que equivalen el 43.9\% demuestran que la frecuencia en la que se emplea la retroalimentación por descubrimiento o reflexión, es "Algunas veces". Y, el $53.2 \%$ de 74 encuestas evidencian que dicho tipo de retroalimentación se da "Siempre". 
Se observa que la mayor frecuencia en la que se emplea la retroalimentación por descubrimiento o reflexión como parte del proceso de enseñanza - aprendizaje del inglés es "Siempre", con un total de 74 encuestas que equivalen al 53.2\% lo que significa que la retroalimentación por descubrimiento o reflexión, se da más de una vez durante las sesiones de aprendizaje en estudiantes de la I.E.S. "José Carlos Mariátegui" Aplicación de la UNA - Puno en el año 2019, en donde los docentes toman como oportunidad de aprendizaje los errores que cometen los estudiantes, les ayudan a analizar las percepciones equivocadas para que finalmente sean ellos quienes comprendan y corrijan su error, realizan varias preguntas constructivas que guían y ayudan a orientar a los estudiantes a comprender y corregir su error, en resumen, los docentes de inglés realizan la retroalimentación por descubrimiento o reflexión para ayudar a los estudiantes a pensar, razonar y reflexionar sobre los errores que cometen durante el proceso de enseñanza aprendizaje.

Tabla 3

La retroalimentación descriptiva y/o adaptativa en la enseñanza - aprendizaje del inglés en estudiantes de la I.E.S. "José Carlos Mariátegui” Aplicación de la UNA - Puno en el año 2019

\begin{tabular}{ccccc}
\hline Valoración & Frecuencia & Porcentaje & Porcentaje válido & Porcentaje acumulado \\
\hline Nunca & 6 & 4.3 & 4.3 & 4.3 \\
Algunas veces & 67 & 48.2 & 48.2 & 52.5 \\
Siempre & 66 & 47.5 & 47.5 & 100.0 \\
Total & 139 & 100.0 & 100.0 & \\
\hline
\end{tabular}

De acuerdo con los datos mostrados en la tabla, se puede decir que, de un total de 139 encuestas aplicadas a estudiantes que representan el 100\%, 6 de estas que equivale al $4.3 \%$ acreditan que la frecuencia en la que se emplea la retroalimentación descriptiva y/o adaptativa como parte del proceso de enseñanza - aprendizaje del inglés, es "Nunca". Por otro lado, 67 encuestan aplicadas que equivalen el 48.2\% demuestran que la frecuencia en la que se emplea la retroalimentación descriptiva y/o adaptativa, es "Algunas veces". Y, el $47.5 \%$ de 66 encuestas evidencian que dicho tipo de retroalimentación se da "Siempre".

Se observa que la mayor frecuencia en la que se emplea la retroalimentación descriptiva y/o adaptativa como parte del proceso de enseñanza - aprendizaje del inglés es "Algunas veces", con un total de 67 encuestas que equivalen al $48.2 \%$ lo que significa que la retroalimentación descriptiva y/o adaptativa, se da al menos una vez durante las sesiones de aprendizaje en estudiantes de la I.E.S. “José Carlos Mariátegui” Aplicación de la UNA - Puno en el año 2019, en donde los docentes de inglés explican detalladamente, emplean diversas formas para ayudar a comprender las malas percepciones del estudiantes, de ser 
el caso readapta su explicación según a las necesidades del estudiante, emplea diversos ejemplos para mejorar la comprensión.

Tabla 4

La retroalimentación elemental en la enseñanza - aprendizaje del inglés en estudiantes de la I.E.S. “José Carlos Mariátegui” Aplicación de la UNA - Puno en el año 2019

\begin{tabular}{ccccc}
\hline Valoración & Frecuencia & Porcentaje & Porcentaje válido & $\begin{array}{c}\text { Porcentaje } \\
\text { acumulado }\end{array}$ \\
\hline Nunca & 10 & 7.2 & 7.2 & 7.2 \\
Algunas veces & 107 & 77.0 & 77.0 & 84.2 \\
Siempre & 22 & 15.8 & 15.8 & 100.0 \\
Total & 139 & 100.0 & 100.0 & \\
\hline
\end{tabular}

De acuerdo con los datos mostrados en la tabla 4, se puede decir que, de un total de 139 encuestas aplicadas a estudiantes que representan el 100\%, 10 de estas que equivale al $7.2 \%$ acreditan que la frecuencia en la que se emplea la retroalimentación elemental como parte del proceso de enseñanza - aprendizaje del inglés, es "Nunca". Por otro lado, 107 encuestan aplicadas que equivalen el $77.0 \%$ demuestran que la frecuencia en la que se emplea la retroalimentación elemental, es “Algunas veces". Y, el 15.8\% de 22 encuestas evidencian que dicho tipo de retroalimentación se da "Siempre".

Se observa que la mayor frecuencia en la que se emplea la retroalimentación elemental como parte del proceso de enseñanza - aprendizaje del inglés es "Algunas veces", con un total de 107 encuestas que equivalen al $77.0 \%$ lo que significa que la retroalimentación elemental, se da al menos una vez durante las sesiones de aprendizaje en estudiantes de la I.E.S. "José Carlos Mariátegui” Aplicación de la UNA - Puno en el año 2019, en donde los docentes ante las malas percepciones $\mathrm{y}$, en efecto, las repuestas equivocadas cometidas por los estudiantes, este solo atina a decirles está bien o simplemente está mal, decirle al estudiante que si está seguro de su respuesta sin brindarle más información, señalarle o decirle la respuesta correcta y cuando el docente solo repite la información o explicaciones dadas en un principio sin readaptarlo a las necesidades de aprendizaje del estudiante.

\section{Contrastación de la hipótesis}

Para contrastar la hipótesis planteada solo se aplicó la media aritmética $(\alpha)$.

Hipótesis: La frecuencia en la que se emplea la retroalimentación como parte del proceso de enseñanza - aprendizaje del inglés es “Algunas veces” en estudiantes de la Institución Educativa Secundaria “José Carlos Mariátegui” Aplicación de la UNA - Puno en el año 2019. El resultado obtenido indica que la frecuencia en la que se emplea la retroalimentación es de “Algunas veces”, alcanzado una media aritmética de 29.7 puntos 
de un total de 139 encuestas aplicadas, lo que significa que la retroalimentación se da al menos una vez, durante el proceso de enseñanza - aprendizaje del inglés.

\section{Discusión}

En la presente investigación se encontró que la retroalimentación se presenta con una frecuencia de algunas veces ya sea la retroalimentación por descubrimiento o reflexión, descriptiva y/o adaptativa y elemental, se da al menos una vez durante las sesiones de aprendizaje en estudiantes. Sin embargo, este resultado contradice a la investigación realizada por, Calvo (2018) quien tuvo como propósito determinar la relación entre la retroalimentación formativa y la comprensión lectora en la I.E. $\mathrm{N}^{\circ} 88024$ de Nuevo Chimbote, obteniendo como respuesta, que no existe una correlación significativa entre las variables de estudio, dado que el 55\% de los docentes no hacen "Nunca"” uso de una retroalimentación formativa, además precisa que el 25\% lo realiza "A veces" y el 20\% "Siempre".

Otro estudio realizado por Uchpas (2020) ha demostrado que la retroalimentación en el aprendizaje de los estudiantes es de nivel medio con un $84 \%$ de estudiantes encuestados, por ello, menciona la necesidad de implementar estrategias de retroalimentación, a través de la capacitación de los docentes. Al respecto, cabe mencionar que la retroalimentación debe ser un soporte que motive y ayude al estudiante a mejorar sus aprendizajes.

Según el propósito específico planteado en esta investigación, conocer la frecuencia en la que emplea la retroalimentación por descubrimiento o reflexiva en el proceso de enseñanza - aprendizaje del inglés en estudiantes de la Institución Educativa Secundaria “José Carlos Mariátegui” Aplicación de la UNA - Puno, se tiene como resultado que este tipo de retroalimentación es la que se emplea frecuentemente con un 53.2\% en la escala de "Siempre". Al respecto, este tipo de retroalimentación es la que se debe brindar a los estudiantes, ya que, les permite a ellos mismos dar respuesta a su problema, a través de preguntas orientadoras y reflexivas que el docente realiza, así como el razonamiento o análisis de casos que se genera en el estudiante (MINEDU, 2017).

Al respecto, Quezada y Salinas (2021) han demostrado que la retroalimentación debe tener influencia en el aprendizaje de lo contrario carecería de significado, en tal sentido, se prioriza la participación de los estudiantes para que interactúen en la construcción de comentarios de calidad.

Así mismo, Samane (2020) ha demostrado que la retroalimentación reflexiva o por descubrimiento que se brinda en la Institución Educativa Juan de Dios Valencia, tiene una correlación positiva media con el aprendizaje autónomo alcanzando un 0,429 y con 
un nivel de significancia de 0.01. Según sus resultados alzados manifiesta que el $66 \%$ estudiantes reciben retroalimentación reflexiva o por descubrimiento, además agrega que este tipo de retroalimentación aumenta el nivel de aprendizaje autónomo en los escolares. Sin embargo, otro estudio realizado por Uchpas (2020) ha concluido que la retroalimentación descriptiva en el aprendizaje de los estudiantes, presenta un nivel medio y deficiente, con un 46,88 \% y 40,63\% respectivamente. Por lo que prioriza la necesidad de incluir estrategias que ayuden y orienten el aprendizaje de los estudiantes, según a sus necesidades particulares.

\section{CONCLUSIONES}

La retroalimentación como parte del proceso de enseñanza - aprendizaje del inglés es "Algunas veces", alcanzando un 52.5\% y con una media aritmética de 29.7 puntos. Es decir, según sea el tipo, por descubrimiento o reflexión, descriptiva y/o adaptativa y elemental se da, al menos una vez, durante las sesiones de aprendizaje en estudiantes de la I.E.S. “José Carlos Mariátegui” Aplicación de la UNA - Puno en el año 2019.

La retroalimentación por descubrimiento o reflexión como parte del proceso de enseñanza - aprendizaje del inglés es "Siempre", con un 53.2\%. Lo cual implica, que se da, más de una vez, durante las sesiones de aprendizaje en estudiantes de la I.E.S. "José Carlos Mariátegui" Aplicación de la UNA - Puno en el año 2019, en donde los docentes crean condiciones favorables como realizar preguntas constructivas que guían y ayudan a los estudiantes a analizar, pensar y reflexionar acerca de las percepciones equivocadas para que, finalmente, sean ellos quienes comprendan y corrijan su error.

La retroalimentación descriptiva y/o adaptativa como parte del proceso de enseñanza aprendizaje del inglés es "Algunas veces", con un 48.2\%. Lo cual implica que se da, al menos una vez, durante las sesiones de aprendizaje en estudiantes de la I.E.S. "José Carlos Mariátegui” Aplicación de la UNA - Puno en el año 2019, en donde los docentes del área de inglés explican detalladamente aquellas deficiencias que se producen durante el aprendizaje y emplean diversas formas (readaptación) para explicar el problema, logrando así comprender y mejorar las malas percepciones de los estudiantes.

La retroalimentación elemental como parte del proceso de enseñanza - aprendizaje del inglés es "Algunas veces", con un 77.0\%. Lo cual implica que se da, al menos una vez, durante las sesiones de aprendizaje en estudiantes de la I.E.S. "José Carlos Mariátegui" Aplicación de la UNA - Puno en el año 2019, en donde los docentes del área de inglés al momento de retroalimentar al estudiante, solo atina a decirle de lo acertado o errado de 
su respuesta, que si está seguro de su respuesta sin mencionarle más información, señalarle la respuesta correcta y cuando solo repite la información o explicaciones, dadas en un principio, sin readaptarlo a las necesidades de aprendizaje del estudiante.

\section{AGRADECIMIENTOS}

A la Universidad Nacional del Altiplano - Puno y a la Facultad de Ciencias de la Educación. A la I. E. S. “José Carlos Mariátegui” Aplicación de la UNA-Puno, por las facilidades brindadas.

\section{REFERENCIAS}

Ašanin, M. (2015). La retroalimentación correctiva entre pares en un intercambio eTándem español-inglés a través de una wiki (tesis doctoral). España: Universidad Autónoma de Madrid. Recuperado de http://hdl.handle.net/10486/672487

Beltran, O. J. (2017). La retroalimentación base fundamental de la evaluación para desarrollar habilidades en el área de inglés (tesis de maestría). Ibague: Universidad del Tolima. Recuperado de https://core.ac.uk/download/pdf/147690288.pdf

Boyco, A. (2019). La retroalimentación en el proceso de aprendizaje de las matemáticas de alumnas de 5to grado de primaria de un colegio privado de Lima (tesis de licenciatura). Lima: Pontificia Universidad Católica del Perú. Recuperado de http://hdl.handle.net/20.500.12404/14051

Caballero, A. B., Negrete, A. J., Arango, L. M. y Jimenez, R. J. (2017). La retroalimentación como estrategia didáctica para fortalecer las prácticas evaluativas en el aula escolar (tesis de maestría). Montería: Universidad Santo Tomás. Recuperado de https://repository.usta.edu.co/bitstream/handle/11634/9513/CaballeroAdelina2017.pdf?sequence $=1$ \&isAllowed $=\mathrm{y}$

Canabal, C., y Margalef, L. (2017). La retroalimentación: la clave para una evaluación orientada al aprendizaje. Profesorado. Revista de Currículum y Formación de Profesorado, 21(2), 149-170. https://www.redalyc.org/articulo.oa?id=56752038009

Calvo, A. (2018). La retroalimentación formativa y la comprensión lectora de la institución educativa $\mathrm{N}^{\circ}$ 88024, Nuevo Chimbote - 2018 (tesis de maestría). Chimbote: Universidad César Vallejo. Recuperado https://repositorio.ucv.edu.pe/bitstream/handle/20.500.12692/36622/Calvo_VTA.pdf?sequence= $1 \&$ isAllowed $=\mathrm{y}$

Charaja, F. (2011). El MAPIC en la metodología de investigación (2da edición). Puno: Impresores Sagitario.

Challco, M. A. (2017). Retroalimentaciones impartidas por los profesores en las clases de la educación física desde la óptica de los estudiantes del VII ciclo de la Institución Educativa Secundaria Gran Unidad Escolar San Carlos de la ciudad de Puno (tesis de licenciatura). Puno: Universidad Nacional del Altiplano.

Flores, M. P. (2015). Aplicación de estrategias de retroalimentación inmediata con uso de dispositivos móviles en la comprensión lectora (tesis de maestría). Santiago: Universidad de Chile. Recuperado de http://repositorio.uchile.cl/handle/2250/137681

Freyre, J. M. (2019). Retroalimentación y aprendizaje del inglés: Prácticas docentes en una IE de Lima Metropolitana (tesis de licenciatura). Lima: Pontificia Universidad Católica del Perú. Recuperado de http://tesis.pucp.edu.pe/repositorio/bitstream/handle/20.500.12404/15615/FREYRE_ALVAREZ _DEL_VILLAR_\%20JANICE_MARITZA.pdf?sequence $=5 \&$ isAllowed $=\mathrm{y}$

Hernández, M. (2017). Usando retroalimentación correctiva automatizada en la enseñanza de la escritura en inglés. Recuperado de www.reibci.org/publicados/2016/dic/2000108.pdf-

Hernández, R., Fernández, C. y Baptista, P. (2014). Metodología de la investigación. 6ta. Edición. México D.F: Mc Graw Hill Interamericana.

Koedinger, K. y Corbett, A. (2006). Cognitive tutors. Technology bringing learning sciences to the classroom. In K. Sawyer (Ed.) The Cambridge handbook of the learning sciences. Nueva York. Cambridge University Press. 
Melmer, R., Burmaster, E. y James, T.K. (2008). Attributes of effective formative assessment. Washington, DC: Council of Chief State School Officers. Recuperado de http://www.ncpublicschools.org/docs/accountability/educators/fastattributes04081.pdf

MINEDU. (2017). Rubricas de observación de aula para la evaluación del desempeño docente. Lima, Perú: Ministerio de Educación Perú.

MINEDU. (2017). Currículo Nacional de la Educación Básica (1ra edición). Lima, Perú: Ministerio de Educación Perú.

Osorio, K. y López, A. (2014). La Retroalimentación Formativa en el Proceso de Enseñanza-Aprendizaje de Estudiantes en Edad Preescolar. Revista Iberoamericana de Evaluación Educativa, 7(1), 13-30. https://dialnet.unirioja.es/descarga/articulo/4704214.pdf

Pino, R. (2007). Metodología de la investigación. San Marcos.

Quezada, S., y Salinas, C. (2021). Modelo de retroalimentación para el aprendizaje: Una propuesta basada en la revisión de literatura. Revista Mexicana de Investigación Educativa, 26(88), 225-251. http://www.scielo.org.mx/pdf/rmie/v26n88/1405-6666-rmie-26-88-225.pdf

Samane, V. A. (2020). La retroalimentación reflexiva o por descubrimiento y su relación con el aprendizaje autónomo de los estudiantes de nivel secundaria de la Institución Educativa Juan de Dios Valencia del distrito de Velille - cusco 2020 (Tesis de licenciatura). Arequipa: Universidad Nacional de San Agustín.

Recuperado

de http://repositorio.unsa.edu.pe/bitstream/handle/20.500.12773/11558/EDsaciva.pdf?sequence=1\& isAllowed $=\mathrm{y}$

Uchpas, J. L. (2020). La retroalimentación en el aprendizaje de los estudiantes de $6^{\circ}$ de primaria de la I.E. 88240 - Nuevo Chimbote, 2020 (Tesis de maestria). Chimbote: Universidad Cesar Vallejo. Recuperado https://repositorio.ucv.edu.pe/bitstream/handle/20.500.12692/52111/Uchpas_BJL\%20$\%$ 20SD.pdf?sequence $=1$

Vergara, M. (2007). Efectos del feedback según niveles de motivación de los aprendices en el aprendizaje de tareas motrices de equilibrio dinámico (Tesis Doctoral). España: Universidad de Granada. Recuperado de http://digibug.ugr.es/bitstream/10481/1818/1/17342156.pdf 